\title{
Children's Imitation Is Affected by Goals, but the Goals Are Outstanding Action Characteristics Rather than Action Outcomes
}

\author{
Takashi Mizuguchi ${ }^{1}$, Ryoko Sugimura ${ }^{2}$, Ryujiro Suzuki ${ }^{1}$, Toshisada Deguchi ${ }^{3}$ \\ ${ }^{1}$ Childfood Education, Iwaki Junior College, Iwaki, Japan; \\ ${ }^{2}$ Psychology, Iwaki Meisei University, Iwaki, Japan; \\ ${ }^{3}$ Comprehensive Educational Science, Tokyo Gakugei University, Tokyo, Japan. \\ Email:mizu-taka@nifty.com \\ Received August 12 ${ }^{\text {nd }}, 2011$; revised September 23 ${ }^{\text {rd }}$, 2011; accepted October $29^{\text {th }}$, 2011.
}

\begin{abstract}
It has been proposed that imitation in children is strongly affected by goals extracted from others' movements. More specifically, imitation-specific goal selection, or a tendency that movement outcomes, rather than means, are more likely to be selected as goals, has been proposed. Conversely, research on imitation in adults has proposed the generalist hypothesis, or the hypothesis that relatively dominant characteristics in movements are simply selected as goals, in the recent years. The present study tested the validity of imitation-specific goal selection with 64 children ( $\mathrm{M}$ age $=5.2$, age range: 4.1 - 6.0) using tasks that were similar to those used in research on imitation in adults. Movements composed of four elements were presented, and errors were analyzed by component. In this process, coloring emphasized one of the four elements, and presentation order of the elements was changed. Results suggested that relatively accentuating a specific element by coloring reduced the errors on the element. In the control condition in which coloring was not applied, the fewest errors were for means. Overall, the results did not support validity of imitation-specific goal selection in children, indicative of the validity the generalist hypothesis.
\end{abstract}

Keywords: Imitation-Specific Goal Selection, Generalist Hypothesis, Children

\section{Introduction}

Imitation plays a central role in acquisition of culture. We inherit cultural products and imagination of the earlier generations through imitation. Furthermore, imitation facilitates communication within a group of the same generation (Tomasello, 1999; Tomasello, Kruger, \& Ratner, 1993). Therefore, learning through imitation is essential in the process of adaptation to a specific culture or a group. Especially, the role that imitation plays is extremely significant for children who are in the process of participating in the culture and group that surround them.

An important research topic concerning the mechanism of imitation is the transformation process from perception to movement. To transform movements of others' that have been visually input into own movements and to express them as an action is the most basic unit of imitation. Several theories and models of the transformation process from perception to movement have been proposed (Rumiati \& Bekkering, 2003). They can be largely grouped into perspectives that support direct and automatic transformation and those that do not. The former group asserts that others' movement is transformed into ones' own directly and automatically as though they communicated with each other. The latter asserts that the transformation process from others' movement to ones' own is mediated by a special processing.

The active intermodal mapping theory (e.g., Meltzoff \& Moore, 1997) can be considered to be a representative view of the former group that supports direct and automatic transformation. Meltzoff et al. argued that even a newborn infant possessed an innate, internal representational system. The authors

*This research was supported by a Grant-in Aid for Japanese Scientific Research (No. 21730532) to the first author. This research was presented at Association for Psychological Science 23rd Annual Convention, Washington, DC, USA, in May 2011. maintained that imitation took place when the other and the self were matched by this system, and other's movements were directly and automatically transformed into one's own movement. However, while imitation of relatively simple movements that Meltzoff addressed, such as sticking out a tongue and making a face, can be easily explained by direct and automatic transformation, imitation of complex movements that humans perform everyday is more difficult to explain.

Perspectives that do not support direct and automatic transformation assert the presence of special processing in the transformation process. The theory of goal-directed imitation (GOADI) maintains that, after examining movements that are more complex than those that were addressed by Meltzoff et al., imitation is affected by goals extracted from the movements (Bekkering, Wohlschläger, \& Gattis, 2000; Gattis, Bekkerning, \& Wohlschläger, 2002; Gleissner, Meltzoff, \& Bekkering, 2000; Wohlschläger, Gattis, \& Bekkerning, 2003). According to GOADI, during the process of imitating, a movement is decomposed into elements of movement patterns, followed by their re-composition. It maintains that the decomposition-recomposition is strongly affected by goals that are extracted from the movement. Out of the elements that compose a series of movement, the elements that are not selected as a goal become less likely to be imitated accurately, often inducing errors, whereas the element that is selected as a goal will attract sufficient attention and be imitated accurately. While GOADI is a theory proposed out of research on children, it is claimed to be applicable to any age group (Wohlschläger, Gattis, \& Bekkerning, 2003).

According to GOADI, selection of goals is biased (e.g., Wohlschläger, Gattis, \& Bekkerning, 2003). Specifically, when a series of movement is presented, the movement outcome, rather than the means, is more likely to be selected. For example, when operation of a machine is presented as a model, the 
action of pressing a switch, instead of the hand that is used or the trajectory of the movement, will be selected as a goal. Therefore, the hand that was used and the trajectory of the movement are less likely to be imitated accurately, whereas and the action of pressing the switch is. Selection of a goal such as this is called imitation-specific goal selection. This differs from the typical processing that is mediated by perception-movement task, and is considered to be a special processing that is observed only in imitation.

However, research on imitation in adults has recently reported a view that does not support imitation-specific goal selection (Bird, Brindley, Leighton, \& Heyes, 2007; Leighton, Bird, \& Heyes, 2010). Bird, Brindley, Leighton, and Heyes (2007) revealed that when an element was relatively accentuated out of various elements that composed a series of movement, that element was likely to be imitated accurately. In the experiment, the task of pen-and-cups, which had been developed as a test of GOADI, was used. For this task, a participant is asked to imitate a movement of holding a pen in a hand and moving the pen closer to a cup in front of him or her. This movement consists of three elements of whether to use the right hand or left (effecter selection), whether to hold the thumb upward or downward while holding a pen (grip selection), and which of the two cups in front to move the pen closer to (object selection). If imitation-specific goal selection, which GOADI maintains, is valid, the outcome is more likely to be imitated accurately than the means is, and thus, the numbers of errors should form a pattern of cup $<$ hand $<$ grip. However, in the experiment of Bird et al., when an element was relatively accentuated by coloring, the number of errors on the accentuated element decreased, breaking the error pattern of cup $<$ hand $<$ grip. In other words, a special processing associated with imitation was not supported, and instead, it was demonstrated that when an element was relatively emphasized in the whole, the movement outcome was not necessarily selected as a goal.

The present study examines the following three points. First is the applicability of the view of Bird et al. with children. As stated earlier, GOADI, which maintains imitation-specific goal selection, was originally proposed based on experiments with children. Therefore, criticism on validity of imitation-specific goal selection is not sufficient if it is based on results of experiments with adults, and it should be built on results of experiments with children, who were originally the primary subject. In addition, Bird et al. have not discussed or tested whether their view is applicable with children. Thus, the present study tested the validity of imitation-specific goal selection by applying tasks that were similar to Bird et al. with children. Next was reexamination of the effects of coloring. Bird et al. analyzed the effects of relative accentuation of one of the three elements of hand selection, grip selection, and selection of a cup. In so doing, they performed coloring on the cup and the hand, but emphasized the grip relatively using a method other than coloring. Hence, the elements that composed the movement were not emphasized by the same method, and thus, their effects could not be compared directly. In contrast, the present study performed coloring by using the same method on all of the elements that composed the movement, and compared their effects directly. Lastly, the present study dealt with the effects of presentation order. Bird et al. compared a case in which elements were presented in the order of the hand selection, grip selection, and the selection of a cup with a case in which elements were presented in the order of the selection of a cup, hand selection, and grip selection. In contrast, in the present study, the number of elements was increased, and the order of component presen- tation was switched more systematically to further analyze the effects of different presentation orders on errors on each elements.

\section{Methods}

\section{Participants}

A total of 64 children ( $\mathrm{M}$ age $=5.2$, age range: $4.1-6.0$ ) participated in the study. They were divided into two groups: a group for ETTE task $(\mathrm{M}$ age $=5.2$, age range $4.1-6.0)$ and a group for TEET task ( $\mathrm{M}$ age $=5.2$, age range $4.1-5.11)$. The mean age and age range of the participants were almost identical with those in the study by Bekkering, Wohlschläger and Gattis (2000) who conducted imitation tasks with children and maintained GOADI initially. Incidentally, it was reported that there was no difference among 4- to 6-years-olds in performance when imitation tasks that were similar to the ones in the present study were conducted with children (Perra \& Gatttis, 2008).

\section{Instruments}

A wooden regular hexahedron $(10 \mathrm{~cm} \times 10 \mathrm{~cm} \times 10 \mathrm{~cm})$, wooden quadrilaterals $(13 \mathrm{~cm} \times 3.5 \mathrm{~cm} \times 3.5 \mathrm{~cm})$, circles made of colored construction paper ( $5 \mathrm{~cm}$ in diameter), and gloves without finger parts was used. The gloves were made of a stretchable material with rubber-like material on the palm part for traction. Coloring was applied on these instruments with white, red, and blue.

\section{Procedure}

The movements that were presented had four elements. They were effecter (hand that was used), tool (instrument that was used), treatment (how to tap), and end point (how to place the instrument). These four elements were systematically combined, and 16 kinds of presentation movements were prepared. That coloring that was applied on each element was summarized in Figure 1 . In all of the conditions, the table top on which the experiment was conducted was a white board. In the control condition, white gloves on two hands, two white tools (quadrilaterals), and a white object (regular hexahedron) were used. In other conditions, coloring was applied on one of the elements in the control condition. In effecter accentuation condition, a red glove on the left hand and a blue glove on the right hand were used. In tool ac centuation condition, a red instrument was placed on the left, while a blue instrument was place on the right. In treatment accentuation condition, the left half of the object was colored in red, while the right half in blue. In end point accentuation condition, a red and a blue circles were placed on the left and right spots on which the instrument was to be placed.

Two tasks were prepared with differing orders of movement presentation. One task was presented in the order of choosing either the right or left hand (effecter), holding either the right or left quadrilateral (tool) with the hand, patting either the right or left end of the object (treatment), and placing the quadrilateral either on the right or left side (end point; ETTE task). The other was a task with the presentation order switched around. First, tool selection was performed by holding either the right or left quadrilateral (tool) as though wrapping it. Next, either the right or left hand (effecter) held the tool, while the other hand leaving the tool, and gently placed the tool either on the right or left side of the object (end point). Finally, without taking the hand off the tool, tapped either the right or left side of the object with the tool (treatment; TEET task). 


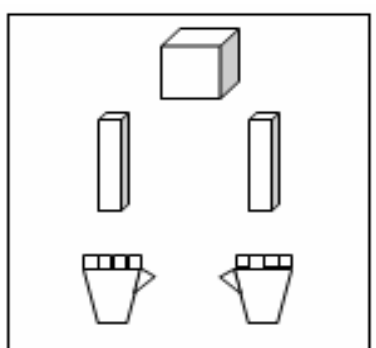

Control Condition

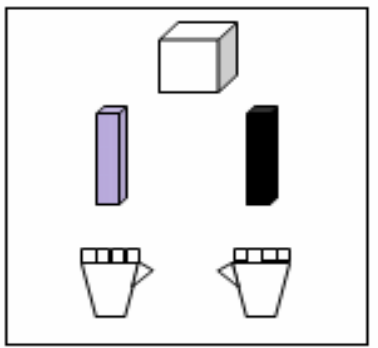

Effecter Accentuation Condition

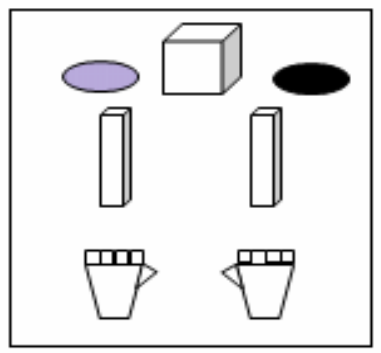

End Point Accentuation Condition

Figure 1.

Placement and coloring of elements in each condition.

Each participant participated in the experiment under all of the conditions. Each participant was asked to imitate only one kind of movement model under each condition to avoid the effects of repeated presentations. The presentation order in each condition was rotated so that there was no bias. Additionally, the movement models were presented so that no movement model of one kind was presented repeatedly under the five conditions executed consecutively. In both tasks, the movement models were presented for approximately $5 \mathrm{~s}$. After presentation, participants were asked to imitate the movement, wearing the same gloves that had been used by the model and using the instruments that were arranged in the same manner as in the model presentation. The imitation responses of the participants were recorded with two video cameras. The presented movements and participants' imitation responses were compared on each element, and agreement and disagreement on the right and left were analyzed. When the response did not match the presented movement, it was judged as an error.

\section{Results}

Figure 2 summarizes errors on each element on each task under each condition. Some elements induced few errors, and normality of distribution and homogeneity of variance were not obtained. Therefore, nonparametric tests were applied for analysis.

First, errors were compared across tasks; errors on one element under one condition were compared across the ETTE task and the TEET task. Mann-Whitney tests yielded no difference across tasks on any element.

Second, errors were compared across conditions, starting with the ETTE task; errors on one element on the ETTE task under the five conditions were compared. Friedman tests resulted significant differences on tool $(\chi 2=38.03, p<.01)$ and end point $(\chi 2=19.79, p<.01)$ on the ETTE task. Signed rank sum tests revealed that for tool, tool accentuation condition < end point accentuation condition $\fallingdotseq$ treatment accentuation condition $\fallingdotseq$ effecter accentuation condition $\fallingdotseq$ control condition $(p<.01)$ and for end point, end point accentuation condition $\fallingdotseq$ tool accentuation condition $<$ control condition $(p<.01)$ and end point accentuation condition $<$ treatment accentuation condition $(p<.01)$. On the TEET task, a significant difference was found with end point $(\chi 2=22.87, p<.01)$. Signed rank tests resulted in a pattern of end point accentuation condition < effecter accentuation condition $\fallingdotseq$ treatment accentuation condition $\fallingdotseq$ control condition $(p<.01)$.

In addition, errors on each element was compared with control condition on each task, in other words, errors on each element under the control condition was paired with errors on the element under other conditions for comparison. Signed rank tests revealed on the ETTE task that tool under the tool accentuation condition, compared to tool under the control condition, induced significantly fewer errors $(Z=-4.58, p<.01)$, end point under the tool accentuation condition, compared to end point under the control condition, induced significantly fewer errors $(Z=-2.71, p<.01)$, and that end point under the end point accentuation condition, compared to end point under the control condition, induced significantly fewer errors $(\mathrm{Z}=-3.21$, $p<.01)$. On the TEET task, it was shown that end point under the end point accentuation condition, compared to end point under the control condition, had induced significantly fewer errors $(Z=-3.21, p<.01)$.

Thirdly, errors were compared across elements. Friedman tests yielded significant differences in the control condition $(\chi 2$ $=35.96, p<.01)$, the effecter accentuation condition $(\chi 2=$ 19.96, $p<.01)$, and the tool accentuation condition $(\chi 2=18.27$, $p<.01)$. Signed rank tests revealed that effecter $<$ tool $\fallingdotseq$ treatment $\fallingdotseq$ end point $(p<.01)$ under the control condition, effecter $<$ tool $\fallingdotseq$ end point $\fallingdotseq$ treatment $(p<.01)$ under the effecter accentuation condition, and that tool $<$ treatment $\fallingdotseq$ end point $\fallingdotseq$ hand $(p<.01)$ under the tool accentuation condition. On the TEET task, there were significant differences in the control condition $(\chi 2=16.52, p<.01)$ and the effecter accentuation condition $(\chi 2=16.02, p<.01)$. Signed rank sum tests revealed that effecter $<$ treatment $\fallingdotseq$ end point $(p<.01)$ under the control condition and effecter $<$ end point $(p<.01)$ under the effecter accentuation condition.

\section{Discussion}

First, we will discuss the validity of imitation-specific goal selection. Wohlschläger, Gattis, and Bekkerning (2003) argued that the outcome was more likely to be imitated accurately when compared to the means, and suggested that there was a special processing associated with imitation that differed from regular processing that mediated perception-movement tasks. For example, they reported that when participants were asked to imitate a movement of using either the right or left hand (ef- 

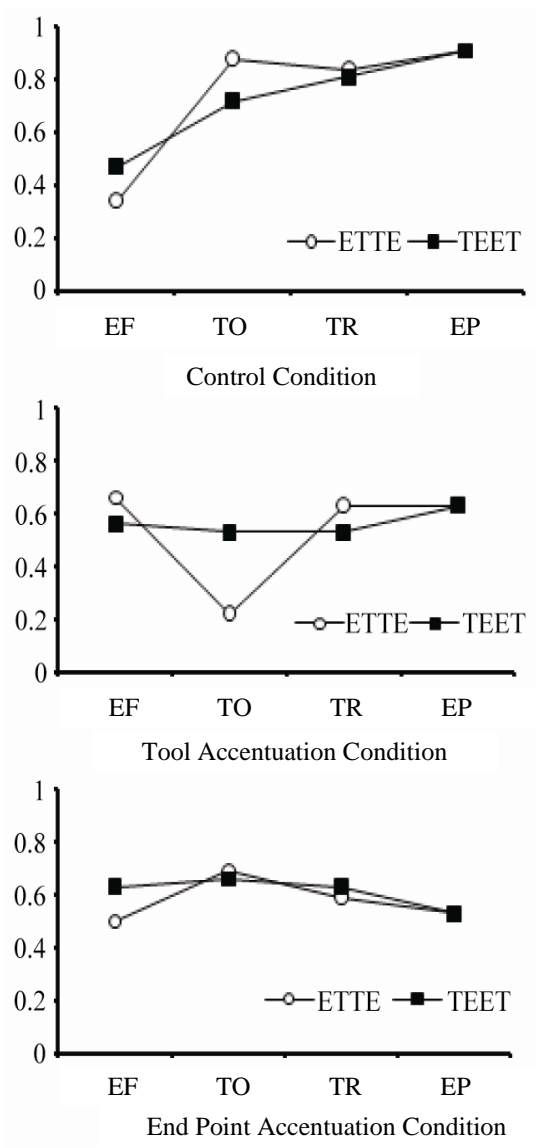

Figure 2.

Errors on elements by condition and task.

fecter selection), directing the thumb upward or downward (grip selection), and choose a cup out of two (object selection), an error pattern of cup < hand < grip resulted. In sum, selection of a cup, which was the outcome of the movement, was seen as the goal and imitated accurately, while the remaining elements were left less likely to be imitated accurately. However, the results of the present study did not support their argument. On the ETTE task under the control condition, hand, which was the effecter, induced fewer errors than any other components did. On the TEET task under the control condition, in other words, when the order of the element presentation was switched around, hand induced the fewest errors also. Put differently, when a regular movement was imitated without emphasis on any elements, the outcome was not imitated any more accurately than the means was. These results are not consistent with the view of imitation-specific goal selection.

Second, we will discuss the effects of coloring. The present study analyzed the effects of accentuating an element that composed a movement by coloring, as did Bird, Brindley, Leighton, and Heyes (2007). The results endorsed effects of coloring. Comparisons of errors across elements showed that on the ETTE task, hand under the effecter accentuation condition and tool under the tool accentuation condition had induced fewer errors. On the TEET task, hand errors under the effecter accentuation condition was shown to be fewer. Further, comparisons of elements across conditions suggested that on both tasks, end point under the end point accentuation condition was fewer. Nonetheless, the effects of coloring were not necessarily clear. As stated earlier, some elements induced fewer errors
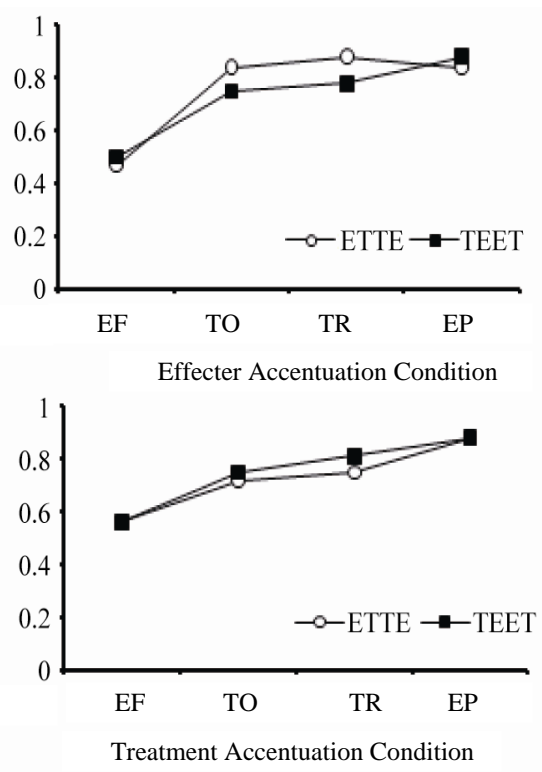

when accentuated with coloring, but some did not induce fewer errors even when accentuated with coloring. The possible reasons for this are as follows.

First possible reason is that coloring affects children and adults differently. Applying a similar method to the method used by Bird et al., the present study attempted to emphasize elements by coloring, but it is possible that its effects on the participating children were not similar to those on adults. Mizuguchi, Suzuki, Sugimura, and Deguchi (2010) directly compared the error patterns of children and adults who each performed imitation tasks that had been designed based on, but a little more complex than, the pen-and-cups task. The results suggested an error pattern that was not different in children and adults although more errors were induced in children than in adults. Judging from this, ease of perceptual processing of each element that composed the movement model, at least, can be considered to be not very different for children and adults. Thus, it can be inferred that the likelihood that coloring affected children and adults differently was low.

Next possibility concerns the effects of the presented movements and the manner of presentation. Surely, the present study applied the method that was similar to the one used by Bird et al., but the presented movements, the manner of presentation, and the method of coloring on elements were not exactly identical. They themselves indicated that imitation was flexible in nature so that depending on how a model was presented, different elements were imitated accurately. Additionally, the research on imitation in children reported that when a movement was presented in a different manner of presentation or 
with a clue associated with imitation, it resulted in a different imitation performance (Mizuguchi, Sugimura, \& Deguchi, 2009; Williamson \& Markman, 2006; Williamson, Meltzoff, \& Markman, 2008). Taking this point into consideration, it is possible that due to some difference in procedures between Bird et al. and the present study, the effects of coloring were not thoroughly revealed. However, there was little difference between error patterns on the ETTE and TEET tasks in the present study. The ETTE and TEET tasks contained the identical elements but in different orders of element presentation, and thus, the impressions that were received from the presented movements were not identical on ETTE and TEET tasks even under the same conditions. If different movements and presentation manners induce different error patterns, as stated above, it is conceivable that error patterns are different between ETTE and TEET tasks. On the contrary, the results of the present study showed that under any conditions, there was no difference in the error patterns between the tasks. Therefore, it should be inferred that the movement and the presentation manner were not the direct cause.

Instead, the fact that coloring did not result in effects similar to Bird et al. can be attributed to the difference in number of imitation trials that were required of the participants. Following Wohlschläger, Gattis, and Bekkering (2003), the participants in the present study were asked to imitate only once under each condition to avoid effects of repeated presentations. In other words, each participant performed imitation only five times. In contrast, in Bird et al., a participant was asked to perform imitation 80 times. The participants presumably gradually grew to pay attention more easily to colored elements as they repeated trials. When imitation was requested only five times in the present study, although the imitation may have been natural and of high ecological validity, the coloring of the elements may not have stood as outstanding as in Bird et al. Thus, it is presumed that coloring did not take effect as easily. If this is a valid interpretation, to obtain the effects that are equivalent to those obtained by Bird et al., it may be necessary to repeat imitation of similar movements that is not very likely to be performed in everyday life approximately 80 consecutive times. Stated otherwise, it is possible that the thorough effects of coloring do not depend on the tasks themselves but on the procedure of the experiment.

Wohlschläger, Gattis, and Bekkerning (2003) proposed imitation-specific goal selection based on the research on imitation primarily in children and maintained that outcomes, rather than means, were more likely to be imitated accurately. This is not observed in the typical perception-movement system, and their perspective suggested the presence of a perception-movement system that was specialized for imitation. On the other hand, Bird et al. reported the results that supported the general hypothesis that simply, parts that relatively stood out were imitated accurately in college students.

The authors argued against the presence of a goal-related, special mechanism in imitation. In contrast, the present study analyzed error patterns in children who performed tasks that were similar to Bird et al. because the experiments that guided the development of the GOADI and imitation-specific goal selection were conducted with children. The results did not endorse the validity of imitation-specific goal selection even in children. Instead, the results were consistent with the general hypothesis that outstanding characteristics of a movement are more likely to be imitated accurately although not exactly identical with the results of Bird et al. Hence, it has been demonstrated that this view is applicable in children as well. Simulta- neously, it has been indicated that a perception-movement system that is specialized for imitation does not exist in children either.

Finally, we will discuss implications for the future. Rumiati and Tessari (2002) revealed that different routes were utilized for processing when the movements to be imitated were meaningless and meaningful, reflecting different availability of existing knowledge. In Wohlschläger, Gattis, and Bekkering (2003) and Bird et al. (2007), participants were asked to imitate operations of everyday tools, such as cups and pens. To the contrary, in the present study, participants were asked to imitate movements that involved solids such as quadrilaterals and a regular hexahedron, instead of everyday tools, and effects of existing knowledge were excluded to the extent possible in order to test the validity of imitation-specific goal selection as purely as possible. If imitation is affected by existing knowledge, it is possible that imitation is affected by knowledge about and familiarity with the materials and tools that were used, as well as the movements. This issue warrants further examination.

Meltzoff (2002) referred to the fact that children are able to imitate movements of adults in spite of the difference in physical size or point of view as one of the problems to be resolved concerning imitation. The present study in combination with Bird et al. have suggested that in at least college students and children, imitation is not performed by a perception-movement system that is specialized for imitation. However, younger children, for example, infants younger than 1 year of age, have not been analyzed, and it is still possible that a special perception-movement system is in place immediately after birth. This issue needs future research as well.

\section{Acknowledgements}

The authors are grateful to the children, parents, and teachers of Iwaki Sakuranbo Nursery School and Iwaki Junior College Attached Kindergarten.

\section{References}

Bekkering, H., Wohlschläger, A., \& Gattis, M. (2000). Imitation of gestures in children is goal-directed. The Quarterly Journal of Experimental Psychology, 53A, 153-164. doi:10.1080/027249800390718

Bird, G., Brindley, R., Leighton, J., \& Heyes, C. (2007). General processes, rather than "Goals", explain imitation errors. Journal of Experimental Psychology: Human Perception and Performance, 33, 1158-1169. doi:10.1037/0096-1523.33.5.1158

Gattis, M., Bekkering, H., \& Wohlschläger, A. (2002). Goal-directed imitation. In A. N. Meltzoff and W. Prinz (Eds.), The imitative mind: Development, evolution, and brain bases (pp. 183-205). New York: Cambridge University Press.

Gleissner, B., Meltzoff, A. N., \& Bekkering, H. (2000). Children's coding of human action: Cognitive factors influencing imitation in 3year-olds. Developmental Science, 3, 405-414. doi:10.1111/1467-7687.00135

Leighton, J., Bird, G., \& Heyes, C. (2010). “Goals” are not an integral component of imitation. Cognition, 114, 423-435. doi:10.1016/j.cognition.2009.11.001

Meltzoff, A. N., \& Moore, M. K. (1997). Explaining facial imitation: A theoretical model. Early Development and Parenting, 6, 179-192. doi:10.1002/(SICI)1099-0917(199709/12)6:3/4<179::AID-EDP157> 3.0.CO;2-R

Meltzoff, A. N. (2002). Elements of a developmental theory of imitation. In A. N. Meltzoff and W. Prinz (Eds.), The imitative mind: Development, evolution, and brain bases (pp. 19-41). New York: Cam- 
bridge University Press.

Mizuguchi, T., Sugimura, R., \& Deguchi, T. (2009). Children's imitations of movements are goal-directed and context-specific. Perceptual and Motor Skills, 108, 513-523. doi:10.2466/pms.108.2.513-523

Mizuguchi, T., Suzuki, R., Sugimura, R., \& Deguchi, T. (2010). Imitation of modeled manipulation of objects by children and adults. Perceptual and Motor Skills, 110, 603-612. doi:10.2466/pms.110.2.603-612

Perra, O., Gattis, M. (2008). Reducing the mapping between perception and action facilitates imitation. British Journal of Developmental Psychology, 26, 133-144. doi:10.1348/026151007X224442

Rumiati, R. I., \& Bekkering, H. (2003). To imitate or not to imitate? How the brain can do it, that is the question! Brain and Cognition, 53, 479-482. doi:10.1016/S0278-2626(03)00208-2

Rumiati, R. I., \& Tessari, A., (2002). Imitation of novel and wellknown actions: The role of short-term memory. Experimental Brain Ressearch, 142, 425-433. doi:10.1007/s00221-002-1070-4
Tomasello, M., Kruger, A. C., \& Ratner, H. H. (1993). Cultural Learning. Behavioral and Brain Science, 16, 495-552. doi:10.1017/S0140525X0003123X

Tomasello, M. (1999). The cultural origins of human cognition. Massachusetts: Harvard University Press.

Williamson, R. A., \& Markman, E. M. (2006). Precision of imitation as a function of preschoolers' understanding of the goal of the demonstaration. Developmental Psychology, 42, 723-731. doi:10.1037/0012-1649.42.4.723

Williamson, R. A., Meltzoff, A. N., \& Markman, E. M. (2008). Prior experiences and perceived efficacy influence 3-year-olds' imitation. Developmental Psychology, 44, 275-285. doi:10.1037/0012-1649.44.1.275

Wohlschläger, A., Garris, M., \& Bekkering, H. (2003). Action generation and action perception in imitation: An instance of the ideomotor principle. Philosophical Transactions: Biological Sciences, 358, 501515. doi:10.1098/rstb.2002.1257 